\title{
Scenario tree reduction for multistage stochastic programs
}

\author{
Holger Heitsch and Werner Römisch \\ Humboldt-University Berlin, Institute of Mathematics, 10099 Berlin, Germany
}

\begin{abstract}
A framework for the reduction of scenario trees as inputs of (linear) multistage stochastic programs is provided such that optimal values and approximate solution sets remain close to each other. The argument is based on upper bounds of the $L_{r}$-distance and the filtration distance, and on quantitative stability results for multistage stochastic programs. The important difference from scenario reduction in two-stage models consists in incorporating the filtration distance. An algorithm is presented for selecting and removing nodes of a scenario tree such that a prescribed error tolerance is met. Some numerical experience is reported.
\end{abstract}

\section{Introduction}

Numerical methods for solving applied stochastic programming models (in finance, production, energy, transportation etc.) mostly rely on approximating the underlying probability distribution by a finitely discrete probability measure. This approximation technique reduces the original infinite-dimensional optimization problem to a finite-dimensional program. To avoid that these optimization problems are too highdimensional, a scenario reduction methodology was suggested in [3] and further developed in $[7,8]$. These scenario reduction methods are based on quantitative stability results for stochastic programs (see the survey [20] and the recent supplement [21] for two-stage models with random recourse) and on the use of distances of probability distributions relying on Monge-Kantorovich mass transportation problems [19]. Although optimal scenario reduction problems are combinatorial optimization models of $k$-median type, and, hence, NP-hard, the forward and backward heuristics suggested in $[3,7]$ and refined in [8] provide encouraging results and are often used in practical applications. The general idea was recently extended in $[12,13]$ to chance constrained and mixed-integer two-stage stochastic programming models.

An important class of stochastic programs for practical applications are models with measurability constraints, e.g., multistage stochastic programs. Recently, the stability behavior of multistage linear stochastic programs was studied in [11]. Its main result states that the distance of optimal values of original and approximate models can be bounded by the $L_{r}$-distance (for some $r \geq 1$ ) and a so-called filtration distance of the underlying stochastic processes. The main computational approach for solving 
multistage models consists in approximating the original stochastic process by a process having finitely many scenarios exhibiting tree structure. Presently, several approaches for the generation of such scenario trees are available. Here, we refer to the survey [2] and to the original papers $[1,9,14,15,16,17,18]$. If such a scenario tree is available, it may again be of interest to reduce it by deleting some of its nodes. Due to the stability behavior of multi-stage models, it is argued in [11, Example 2.7] that scenario tree reduction in multistage models should be based on $L_{r}$-distances as well as on filtration distances.

In this paper, we take up the latter issue and develop a sound theoretical basis for scenario tree reduction in multistage stochastic programming models. To do so, we review stability results for the multistage situation (in Section 2) and derive new bounds for both the $L_{r}$-distance and the filtration distances between a scenario tree and its reduced version (in Section 3). These bounds motivate algorithms for reducing scenario trees. In Section 4 we present a specific algorithm based on recursive single node reduction. In Section 5 we report on numerical experience of the tree reduction algorithm and show that its outcomes strongly depends on the use of both types of distances, namely, the $L_{r}$-distance and the filtration distance. In particular, the results indicate that applying the scenario reduction techniques from $[7,8]$ (i.e., methods that are only based on the $L_{r}$-distance) to the multistage situation is not appropriate.

\section{A review of stability in multistage stochastic pro- gramming}

Let $\xi=\left\{\xi_{t}\right\}_{t=1}^{T}$ be a stochastic process defined on some probability space $(\Omega, \mathcal{F}, \mathbb{P})$ and with $\xi_{t}$ taking values in $\mathbb{R}^{d}$. It is assumed that this process enters an optimization model and that the (stochastic) decision $x_{t}$ at $t$ maps from $\Omega$ to $\mathbb{R}^{m_{t}}$ is nonanticipative, i.e., depends only on $\xi^{t}:=\left(\xi_{1}, \ldots, \xi_{t}\right)$. The latter property is equivalent to the measurability constraint stating that $x_{t}$ is measurable with respect to the $\sigma$-field $\mathcal{F}_{t}(\xi) \subseteq \mathcal{F}$ generated by $\xi^{t}$. We assume that $\xi_{1}$ is deterministic, i.e., that $\mathcal{F}_{1}(\xi)=\{\emptyset, \Omega\}$. Then the stochastic process $\xi$ is accompanied by a filtration $\left(\mathcal{F}_{t}(\xi)\right)_{t=1}^{T}$ of $\sigma$-fields satisfying

$$
\mathcal{F}_{1}(\xi)=\{\emptyset, \Omega\} \subseteq \cdots \subseteq \mathcal{F}_{t}(\xi) \subseteq \mathcal{F}_{t+1}(\xi) \subseteq \cdots \subseteq \mathcal{F}_{T}(\xi) \subseteq \mathcal{F}
$$

We consider the linear multistage stochastic programming model

$$
\min \left\{\mathbb{E}\left[\sum_{t=1}^{T}\left\langle b_{t}\left(\xi_{t}\right), x_{t}\right\rangle\right] \mid \begin{array}{l}
x_{t} \in X_{t} \\
x_{t} \text { is } \mathcal{F}_{t}(\xi) \text {-measurable, } t=1, \ldots, T \\
A_{t, 0} x_{t}+A_{t, 1}\left(\xi_{t}\right) x_{t-1}=h_{t}\left(\xi_{t}\right), t=2, \ldots, T
\end{array}\right\}
$$

where the subsets $X_{t}$ of $\mathbb{R}^{m_{t}}$ are nonempty and polyhedral, the cost coefficients $b_{t}\left(\xi_{t}\right)$ belong to $\mathbb{R}^{m_{t}}$, the right-hand sides $h_{t}\left(\xi_{t}\right)$ are in $\mathbb{R}^{n_{t}}, A_{t, 0} \in \mathbb{R}^{n_{t} \times m_{t}}$ are fixed recourse matrices and $A_{t, 1}\left(\xi_{t}\right) \in \mathbb{R}^{n_{t} \times m_{t-1}}$ technology matrices, respectively. We assume that costs $b_{t}(\cdot)$, right-hand sides $h_{t}(\cdot)$ and technology matrices $A_{t, 1}(\cdot)$ depend affinely on $\xi_{t}$ covering the situation that some of the components of $b_{t}$ and $h_{t}$, and of the elements of $A_{t, 1}$ are random. Note that the two constraints $x_{t} \in X_{t}$ and $A_{t, 0} x_{t}+A_{t, 1}\left(\xi_{t}\right) x_{t-1}=h_{t}\left(\xi_{t}\right)$ mean $x_{t}(\omega) \in X_{t}$ and $A_{t, 0} x_{t}(\omega)+A_{t, 1}\left(\xi_{t}(\omega)\right) x_{t-1}(\omega)=h_{t}\left(\xi_{t}(\omega)\right)$ for $\mathbb{P}$-almost every $\omega \in \Omega$. 
In addition to the pointwise constraint with probability 1, measurability, filtration or information constraints appear in (1). They are functional and non-pointwise at least if $T>2$ and $\mathcal{F}_{1}(\xi) \varsubsetneqq \mathcal{F}_{t}(\xi) \varsubsetneqq \mathcal{F}_{T}(\xi)$ for some $1<t<T$. The presence of such qualitatively different constraints constitutes the origin of both the theoretical and computational challenges of multistage models.

Next we record results of the recent papers $[11,9]$. We assume that the stochastic input process $\xi$ belongs to the Banach space $L_{r}\left(\Omega, \mathcal{F}, \mathbb{P} ; \mathbb{R}^{s}\right)$ with $s:=T d$ and $r \geq$ 1. The multistage model (1) is regarded as an optimization problem in the space $L_{r^{\prime}}\left(\Omega, \mathcal{F}, \mathbb{P} ; \mathbb{R}^{m}\right)$ with $m=\sum_{t=1}^{T} m_{t}$ and endowed with the norm

$$
\|x\|_{r^{\prime}}:=\left(\sum_{t=1}^{T} \mathbb{E}\left[\left|x_{t}\right|^{r^{\prime}}\right]\right)^{\frac{1}{r^{\prime}}}\left(1 \leq r^{\prime}<\infty\right) \quad \text { or } \quad\|x\|_{\infty}:=\max _{t=1, \ldots, T} \operatorname{ess} \sup \left|x_{t}\right|,
$$

where the number $r^{\prime}$ is defined by

$$
r^{\prime}:=\left\{\begin{array}{cl}
\frac{r}{r-1} & , \text { if only costs are random } \\
r & , \text { if only right-hand sides are random } \\
r=2 & , \text { if only costs and right-hand sides are random } \\
\infty & , \text { if all technology matrices are random and } r \geq T .
\end{array}\right.
$$

The choice of $r$ and the definition of $r^{\prime}$ are motivated by the knowledge on existing moments of the input process and by having the stochastic program well defined (in particular, such that $\left\langle b_{t}\left(\xi_{t}\right), x_{t}\right\rangle$ is integrable for every decision $x$ and $\left.t=1, \ldots, T\right)$.

Next we need to introduce some notations. Let $F$ denote the objective function defined on $L_{r}\left(\Omega, \mathcal{F}, \mathbb{P} ; \mathbb{R}^{s}\right) \times L_{r^{\prime}}\left(\Omega, \mathcal{F}, \mathbb{P} ; \mathbb{R}^{m}\right) \rightarrow \mathbb{R}$ by $F(\xi, x):=\mathbb{E}\left[\sum_{t=1}^{T}\left\langle b_{t}\left(\xi_{t}\right), x_{t}\right\rangle\right]$, let

$$
\mathcal{X}_{t}\left(x_{t-1} ; \xi_{t}\right):=\left\{x_{t} \in X_{t} \mid A_{t, 0} x_{t}+A_{t, 1}\left(\xi_{t}\right) x_{t-1}=h_{t}\left(\xi_{t}\right)\right\}
$$

denote the $t$-th feasibility set for every $t=2, \ldots, T$ and

$$
\begin{aligned}
& \mathcal{X}(\xi):=\left\{x=\left(x_{1}, x_{2}, \ldots, x_{T}\right) \in \times_{t=1}^{T} L_{r^{\prime}}\left(\Omega, \mathcal{F}_{t}(\xi), \mathbb{P} ; \mathbb{R}^{m_{t}}\right) \mid\right. \\
& \left.x_{1} \in X_{1}, x_{t} \in \mathcal{X}_{t}\left(x_{t-1} ; \xi_{t}\right)\right\}
\end{aligned}
$$

the set of feasible elements of (1) with input $\Xi$. Then the multistage stochastic program (1) may be rewritten as

$$
\min \{F(\xi, x): x \in \mathcal{X}(\xi)\} .
$$

Furthermore, let $v(\xi)$ denote its optimal value and, for any $\alpha \geq 0$,

$$
S_{\alpha}(\xi):=\{x \in \mathcal{X}(\xi): F(\xi, x) \leq v(\xi)+\alpha\} \quad \text { and } \quad S(\xi):=S_{0}(\xi)
$$

denote the $\alpha$-approximate solution set and the solution set of the stochastic program (3) with input $\xi$, respectively.

The following conditions are imposed on (3):

(A1) $\xi \in L_{r}\left(\Omega, \mathcal{F}, \mathbb{P} ; \mathbb{R}^{s}\right)$, i.e., $\int_{\Omega}|\xi(\omega)|^{r} d \mathbb{P}(\omega)<\infty$, for some $r \geq 1$.

(A2) There exists a $\delta>0$ such that for any $\tilde{\xi} \in L_{r}\left(\Omega, \mathcal{F}, \mathbb{P} ; \mathbb{R}^{s}\right)$ with $\|\tilde{\xi}-\xi\|_{r} \leq \delta$, any $t=2, \ldots, T$ and any $x_{1} \in \mathcal{X}_{1}\left(\tilde{\xi}_{1}\right), x_{\tau} \in \mathcal{X}_{\tau}\left(x_{\tau-1} ; \tilde{\xi}_{\tau}\right), \tau=2, \ldots, t-1$, there exists an $\mathcal{F}_{t}(\tilde{\xi})$-measurable $x_{t} \in \mathcal{X}_{t}\left(x_{t-1} ; \tilde{\xi}_{t}\right)$ (relatively complete recourse locally around $\xi$ ). 
(A3) The optimal values $v(\tilde{\xi})$ of (3) with input $\tilde{\xi}$ are finite for all $\tilde{\xi}$ in a neighborhood of $\xi$ and the objective function $F$ is level-bounded locally uniformly at $\xi$, i.e., for some $\alpha>0$ there exist a constant $\delta>0$ and a bounded subset $B$ of $L_{\infty}\left(\Omega, \mathcal{F}, \mathbb{P} ; \mathbb{R}^{m}\right)$ such that $S_{\alpha}(\tilde{\xi})$ is contained in $B$ for all $\tilde{\xi} \in L_{r}\left(\Omega, \mathcal{F}, \mathbb{P} ; \mathbb{R}^{s}\right)$ with $\|\tilde{\xi}-\xi\|_{r} \leq \delta$.

The following stability result states that multistage models behave stable at some stochastic input process if both its probability distribution and its filtration are approximated simultaneously in terms of the $L_{r}$-distance and of one of the filtration distances

$$
\begin{aligned}
D_{\mathrm{f}, \infty}(\xi, \tilde{\xi}) & :=\sup _{\|x\|_{\infty} \leq 1} \sum_{t=2}^{T-1}\left\|\mathbb{E}\left[x_{t} \mid \mathcal{F}_{t}(\xi)\right]-\mathbb{E}\left[x_{t} \mid \mathcal{F}_{t}(\tilde{\xi})\right]\right\|_{r^{\prime}} \\
D_{\mathrm{f}, \infty}^{*}(\xi, \tilde{\xi}) & :=\sup _{\|x\|_{\infty} \leq 1} \sum_{t=2}^{T}\left\|\mathbb{E}\left[x_{t} \mid \mathcal{F}_{t}(\xi)\right]-\mathbb{E}\left[x_{t} \mid \mathcal{F}_{t}(\tilde{\xi})\right]\right\|_{r^{\prime}}
\end{aligned}
$$

where $\mathcal{F}_{t}(\xi)$ and $\mathcal{F}_{t}(\tilde{\xi})$ denote the $\sigma$-fields generated by $\xi^{t}$ and $\tilde{\xi}^{t}$, respectively, and $\mathbb{E}\left[\cdot \mid \mathcal{F}_{t}(\xi)\right]$ and $\mathbb{E}\left[\cdot \mid \mathcal{F}_{t}(\tilde{\xi})\right]$ the corresponding conditional expectations.

Theorem 2.1 Let (A1), (A2) and (A3) be satisfied and $X_{1}$ be bounded.

Then there exist positive constants $L$ and $\delta$ such that the estimate

$$
|v(\xi)-v(\tilde{\xi})| \leq L\left(\|\xi-\tilde{\xi}\|_{r}+D_{\mathrm{f}, \infty}(\xi, \tilde{\xi})\right)
$$

holds for all random elements $\tilde{\xi} \in L_{r}\left(\Omega, \mathcal{F}, \mathbb{P} ; \mathbb{R}^{s}\right)$ with $\|\tilde{\xi}-\xi\|_{r} \leq \delta$.

Furthermore, if the solution sets $S(\xi)$ and $S(\tilde{\xi})$ are nonempty, there exist $\bar{L}>0$ and $\bar{\varepsilon}>0$ such that the estimate

$$
d d_{\infty}\left(S_{\varepsilon}(\xi), S_{\varepsilon}(\tilde{\xi})\right) \leq \frac{\bar{L}}{\varepsilon}\left(\|\xi-\tilde{\xi}\|_{r}+D_{\mathrm{f}, \infty}^{*}(\xi, \tilde{\xi})\right)
$$

holds for any $\varepsilon \in(0, \bar{\varepsilon})$. Here, $d_{\infty}$ denotes the Pompeiu-Hausdorff distance of bounded subsets of $L_{r^{\prime}}$.

The first part of Theorem 2.1 is essentially [11, Theorem 2.1], where compared to [11], condition (A3) allows to make use of the filtration distances $D_{\mathrm{f}, \infty}$ or $D_{\mathrm{f}, \infty}^{*}$ (cf. the discussion in [9, Section 3]). The second part of Theorem 2.1 is proved in [10].

Finally, we mention that Theorem 2.1 remains valid if the expectation $\mathbb{E}$ in the objective of (1) is replaced by a multi-period polyhedral risk functional satisfying a certain uniform level boundedness property (see [6]). Multi-period polyhedral risk functionals and their incorporation into multi-stage stochastic programming models are studied in [4].

\section{Bounding the $L_{r}$-minimal and filtration distance}

Let $\xi=\left\{\xi_{t}\right\}_{t=1}^{T}$ be a stochastic process on the probability space $(\Omega, \mathcal{F}, \mathbb{P})$ having a finite number of scenarios $\xi^{i}$ with probabilities $p_{i}, i=1, \ldots, N$, in form of a scenario tree. Let $I_{t}$ denote the index set of realizations of $\xi_{t}$. If we set $A_{t i}:=\xi_{t}^{-1}\left(\left\{\xi_{t}^{i}\right\}\right)$ for every $i \in I_{t}$, the system $\left\{A_{t i}\right\}_{i \in I_{t}}$ is a partition of $\Omega$ and generates the $\sigma$-field $\mathcal{F}_{t}(\xi)$. 
We set $p_{t}^{i}:=\mathbb{P}\left(A_{t i}\right), i \in I_{t}, t=1, \ldots, T$, and have, in particular, that $I_{T}=\{1, \ldots, N\}$ and $p_{T}^{i}=p_{i}$ for every $i \in I_{T}$. Furthermore, we have

$$
\xi_{t}=\sum_{i \in I_{t}} \xi_{t}^{i} \mathbb{1}_{A_{t i}} \quad(t=1, \ldots, T)
$$

where $\mathbb{1}_{A}$ denotes the characteristic function of a subset $A$ of $\Omega$. The tree structure of $\xi$ implies that $I_{1}$ is a singleton and that

$$
I_{1} \subseteq I_{2} \subseteq \cdots \subseteq I_{t} \subseteq I_{t+1} \subseteq \cdots \subseteq I_{T}=\{1, \ldots, N\}
$$

holds. Moreover, if $I_{t, i} \subseteq I_{t+1}$ denotes the index set of successors to $\xi_{t}^{i}$ at $t+1$, the relations

$$
p_{t}^{i}=\sum_{j \in I_{t, i}} p_{t+1}^{j} \quad\left(i \in I_{t}\right)
$$

are valid for every $t=1, \ldots, T-1$. Any node of the tree corresponds to a pair $(t, i) \in\{1, \ldots, T\} \times I_{t}$.

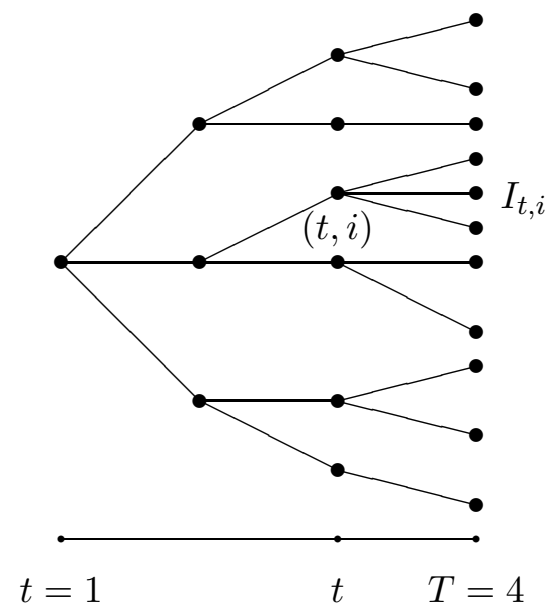

Figure 1: Scenario tree $\xi$ with $\left|I_{t}\right|=6,\left|I_{t, i}\right|=3$ and $\left|I_{T}\right|=11$

Now, let $\xi^{\text {red }}$ be a stochastic process on $(\Omega, \mathcal{F}, \mathbb{P})$ that we regard as reduced scenario tree obtained from $\xi$. If $I_{t}^{\text {red }}$ denotes the index set of realizations of $\xi_{t}^{\text {red }}$, the latter means

$$
I_{1}^{\mathrm{red}}=I_{1} \quad \text { and } \quad I_{t}^{\mathrm{red}} \subseteq I_{t} \quad(t=2, \ldots, T),
$$

where at least for some $t \in\{2, \ldots, T\}$ we have $I_{t}^{\text {red }} \subset I_{t}$. Let $\xi^{j, \text { red }}, j \in I_{T}^{\text {red }}$, denote the scenarios of $\xi^{\text {red }}$. Let us further denote by $J_{t}:=I_{t} \backslash I_{t}^{\text {red }}$ the index set of all withdrawn realizations at time $t$ and by $E_{t j}$ the set $E_{t j}:=\left(\xi_{t}^{\text {red }}\right)^{-1}\left(\xi_{t}^{j}\right)$ for every $j \in I_{t}^{\text {red }}$. The system $\left\{E_{t j}\right\}_{j \in I_{t}^{\text {red }}}$ forms a partition of $\Omega$ and generates the $\sigma$-field $\mathcal{F}_{t}\left(\xi^{\text {red }}\right)$. Moreover, for every $j \in I_{t}^{\text {red }}$, let $J_{t, j} \subset I_{t}$ denote the index set such that $\xi_{t}^{i}=\xi_{t}^{j}=\xi_{t}^{j, \text { red }}$ holds for all $i \in J_{t, j}$, i.e., the index set of all scenarios in $I_{t}$ which have been identified with $\xi_{t}^{j}$ during the reduction process. The index sets $\left\{J_{t, j}\right\}_{j \in I_{t}^{\text {red }}}$ form a partition of $I_{t}$ and it holds

$$
\begin{aligned}
E_{t j} & =\bigcup_{i \in J_{t, j}} A_{t i} \\
\pi_{t}^{j} & :=\mathbb{P}\left(E_{t j}\right)=\sum_{i \in J_{t, j}} p_{t}^{i} \quad\left(j \in I_{t}^{\mathrm{red}}\right) .
\end{aligned}
$$




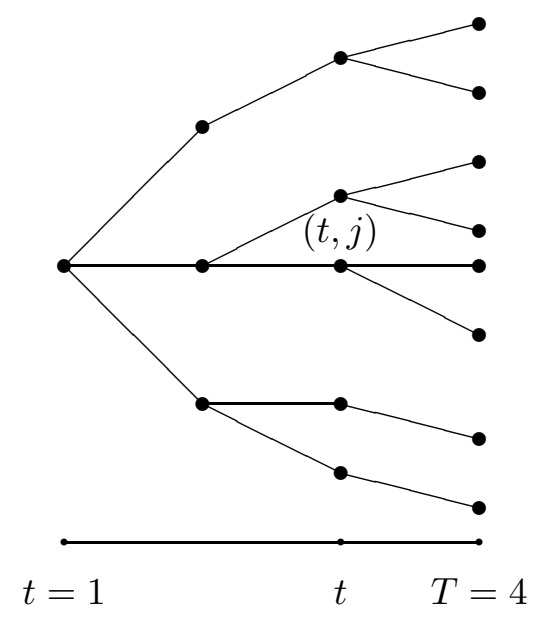

Figure 2: Scenario tree $\xi^{\text {red }}$ with $\left|I_{t}^{\text {red }}\right|=5,\left|J_{t, j}\right|=2$ and $\left|I_{T}^{\text {red }}\right|=8$

If the assumptions of Theorem 2.1 are satisfied for some $1 \leq r<+\infty$, the distances of optimal values and $\varepsilon$-approximate solution sets get small if both distances

$$
\left\|\xi-\xi^{\mathrm{red}}\right\|_{r} \quad \text { and } \quad D_{\mathrm{f}, \infty}^{*}\left(\xi, \xi^{\mathrm{red}}\right)
$$

are small. Hence, if a tolerance $\varepsilon>0$ is given, it is reasonable to require

$$
w_{1}\left\|\xi-\xi^{\mathrm{red}}\right\|_{r}+w_{2} D_{\mathrm{f}, \infty}^{*}\left(\xi, \xi^{\mathrm{red}}\right) \leq \varepsilon,
$$

where $w_{i}, i=1,2$, denote positive weighting factors such that $w_{1}\left\|\xi-\xi^{\text {red }}\right\|_{r}$ and $w_{2} D_{\mathrm{f}, \infty}^{*}\left(\xi, \xi^{\text {red }}\right)$ belong to $(0,1]$.

The condition (9) appears as a natural condition for reducing the scenario tree $\xi$. A canonical choice for the factors $w_{1}$ and $w_{2}$ is obtained by selecting $i^{*} \in I_{T}$ such that the corresponding scenario $\xi^{i^{*}}$ represents the best approximation of $\xi$ with respect to the $L_{r}$-distance. More precisely, if $\xi^{*}$ denotes the corresponding deterministic scenario process, i.e., $\xi^{*}(\omega)=\xi^{i^{*}}$, for all $\omega \in \Omega$, we have

$$
\left\|\xi-\xi^{*}\right\|_{r} \leq\left\|\xi-\tilde{\xi}^{*}\right\|_{r}
$$

for all deterministic processes $\tilde{\xi}^{*}$ consisting of only one given scenario, i.e., for all processes with $\tilde{\xi}^{*}(\omega)=\xi^{i}$, for all $\omega \in \Omega$, where $i \in I_{T}$. Then the weighting factors are defined by

$$
w_{1}=\frac{1}{\left\|\xi-\xi^{*}\right\|_{r}} \quad \text { and } \quad w_{2}=\frac{1}{D_{\mathrm{f}, \infty}^{*}\left(\xi, \xi^{*}\right)} .
$$

Next we derive bounds for both distances in (9). They are of the form

$$
\begin{aligned}
\left\|\xi-\xi^{\mathrm{red}}\right\|_{r} & =\left(\sum_{t=2}^{T} \mathbb{E}\left[\left|\xi_{t}-\xi_{t}^{\mathrm{red}}\right|^{r}\right]\right)^{\frac{1}{r}}, \\
D_{\mathrm{f}, \infty}^{*}\left(\xi, \xi^{\mathrm{red}}\right) & =\sum_{t=2}^{T} \sup _{\substack{x_{t} \in L_{\infty}\left(\mathcal{F}_{t}(\xi)\right) \\
\left\|x_{t}\right\| \infty \leq 1}}\left\|x_{t}-\mathbb{E}\left[x_{t} \mid \mathcal{F}_{t}\left(\xi^{\mathrm{red}}\right)\right]\right\|_{r^{\prime}},
\end{aligned}
$$


where $L_{\infty}\left(\mathcal{F}_{t}(\xi)\right)=L_{\infty}\left(\Omega, \mathcal{F}_{t}(\xi), \mathbb{P} ; \mathbb{R}^{m_{t}}\right)$. The latter formula is a consequence of the identity

$$
\begin{aligned}
D_{\mathrm{f}, \infty}^{*}\left(\xi, \xi^{\mathrm{red}}\right) & =\sup _{\|x\|_{\infty} \leq 1} \sum_{t=2}^{T}\left\|\mathbb{E}\left[x_{t} \mid \mathcal{F}_{t}(\xi)\right]-\mathbb{E}\left[x_{t} \mid \mathcal{F}_{t}\left(\xi^{\mathrm{red}}\right)\right]\right\|_{r^{\prime}} \\
& =\sup _{\|x\|_{\infty} \leq 1} \sum_{t=2}^{T}\left\|\mathbb{E}\left[x_{t} \mid \mathcal{F}_{t}(\xi)\right]-\mathbb{E}\left[\mathbb{E}\left[x_{t} \mid \mathcal{F}_{t}(\xi)\right] \mid \mathcal{F}_{t}\left(\xi^{\mathrm{red}}\right)\right]\right\|_{r^{\prime}} \\
& =\sup _{\substack{\|x\|_{\infty} \leq 1 \\
x_{t} \in L_{\infty}\left(\mathcal{F}_{t}(\xi)\right)}} \sum_{t=2}^{T}\left\|x_{t}-\mathbb{E}\left[x_{t} \mid \mathcal{F}_{t}\left(\xi^{\mathrm{red}}\right)\right]\right\|_{r^{\prime}}
\end{aligned}
$$

which is due to the inclusion $\mathcal{F}_{t}\left(\xi^{\text {red }}\right) \subseteq \mathcal{F}_{t}(\xi)$, and the fact that the condition $\|x\|_{\infty} \leq 1$ is equivalent to $\left\|\mathbb{E}\left[x_{t} \mid \mathcal{F}_{t}(\xi)\right]\right\|_{\infty} \leq 1$ for every $t=1, \ldots, T$.

To derive explicit expressions for (12), we use the measurability of $x_{t}$ with respect to $\mathcal{F}_{t}\left(\xi^{\text {red }}\right)$ and denote the scenarios $x_{t}$ by $x_{t}^{i}, i \in I_{t}$, for every $t=2, \ldots, T$. We obtain for the conditional expected values

$$
\mathbb{E}\left[x_{t} \mid E_{t j}\right]=\frac{\int_{E_{t j}} x_{t} \mathbb{P}(d \omega)}{\mathbb{P}\left(E_{t j}\right)}=\frac{1}{\pi_{t}^{j}} \sum_{i \in J_{t, j}} p_{t}^{i} x_{t}^{i}
$$

for every $j \in I_{t}^{\text {red }}$ and $t=2, \ldots, T$. For $1 \leq r^{\prime}<\infty$ we get from (12)

$$
D_{\mathrm{f}, \infty}^{*}\left(\xi, \xi^{\mathrm{red}}\right)=\sum_{t=2}^{T} \sup _{\left\|x_{t}\right\|_{\infty} \leq 1}\left(\mathbb{E}\left[\left|\sum_{i \in I_{t}} x_{t}^{i} \mathbb{1}_{A_{t i}}-\sum_{j \in I_{t}^{\mathrm{red}}} \mathbb{E}\left[x_{t} \mid E_{t j}\right] \mathbb{1}_{E_{t j}}\right|^{r^{\prime}}\right]\right)^{\frac{1}{r^{\prime}}}
$$

and continue

$$
\begin{aligned}
D_{\mathrm{f}, \infty}^{*}\left(\xi, \xi^{\mathrm{red}}\right) & =\sum_{t=2}^{T} \sup _{\left\|x_{t}\right\|_{\infty} \leq 1}\left(\mathbb{E}\left[\left|\sum_{j \in I_{t}^{\text {red }}} \sum_{i \in J_{t, j}} x_{t}^{i} \mathbb{1}_{A_{t i}}-\sum_{j \in I_{t}^{\text {red }}} \mathbb{E}\left[x_{t} \mid E_{t j}\right] \sum_{i \in J_{t, j}} \mathbb{1}_{A_{t i}}\right|^{r^{\prime}}\right]\right)^{\frac{1}{r^{\prime}}} \\
& =\sum_{t=2}^{T} \sup _{\left\|x_{t}\right\|_{\infty} \leq 1}\left(\sum_{j \in I_{t}^{\text {red }}} \sum_{i \in J_{t, j}} p_{t}^{i}\left|x_{t}^{i}-\frac{1}{\pi_{t}^{j}} \sum_{k \in J_{t, j}} p_{t}^{k} x_{t}^{k}\right|^{r^{\prime}}\right)^{\frac{1}{r^{\prime}}}
\end{aligned}
$$

\subsection{The $L_{r}$-distance}

Now we start to discuss the $L_{r}$-distance $\left\|\xi-\xi^{\text {red }}\right\|_{r}$ between the two processes $\xi$ and $\xi^{\text {red }}$. According to our notations we directly obtain from (11)

$$
\begin{aligned}
\left\|\xi-\xi^{\mathrm{red}}\right\|_{r}^{r} & =\sum_{t=2}^{T} \int_{\Omega}\left|\xi_{t}-\xi_{t}^{\mathrm{red}}\right|^{r}=\sum_{t=2}^{T} \sum_{j \in I_{t}^{\mathrm{red}}} \sum_{i \in J_{t, j}} \int_{A_{t i}}\left|\xi_{t}-\xi_{t}^{\mathrm{red}}\right|^{r} \\
& =\sum_{t=2}^{T} \sum_{j \in I_{t}^{\mathrm{red}}} \sum_{i \in J_{t, j}} p_{t}^{i}\left|\xi_{t}^{i}-\xi_{t}^{j}\right|^{r}
\end{aligned}
$$


This means that the $L_{r}$-distance between a given process and a reduced one depends on the probabilities of all withdrawn scenario components and on their distances to some of the remaining scenario components.

\subsection{The filtration distance}

Next we derive an estimate for $D_{\mathrm{f}, \infty}^{*}\left(\xi, \xi^{\text {red }}\right)$ given by (13) in case of $r^{\prime}<\infty$ and $\left\|x_{t}\right\|_{\infty}:=\max _{i \in I_{t}}\left|x_{t}^{i}\right|$ for every $x_{t} \in L_{\infty}\left(\mathcal{F}_{t}(\xi)\right)$.

Proposition 3.1 Consider the $\ell_{r^{\prime}}$-norms

$$
\left|y_{t}\right|:=\left(\sum_{s=1}^{m_{t}}\left|y_{t, s}\right|^{r^{\prime}}\right)^{\frac{1}{r^{\prime}}}
$$

in $\mathbb{R}^{m_{t}}$ for every $t=2, \ldots, T$. Then we have

$$
D_{\mathrm{f}, \infty}^{*}\left(\xi, \xi^{\mathrm{red}}\right) \leq \max _{t=2, \ldots, T} m_{t}^{\frac{1}{r^{\prime}}} \sum_{t=2}^{T}\left(\sum_{j \in I_{t}^{\mathrm{red}}} \max \left\{f_{t, r^{\prime}}^{j}\left(\sum_{i \in J} p_{t}^{i}\right): J \subset J_{t, j}\right\}\right)^{\frac{1}{r^{\prime}}}
$$

where the function $f_{t, r^{\prime}}^{j}$ is defined by $f_{t, r^{\prime}}^{j}(p):=\frac{\left(2 p\left(\pi_{t}^{j}-p\right)^{r^{\prime}}+2\left(\pi_{t}^{j}-p\right) p^{r^{\prime}}\right)}{\left(\pi_{t}^{j}\right)^{r^{\prime}}}$ for every $p \in\left[0, \pi_{t}^{j}\right]$, $j \in I_{t}^{\mathrm{red}}, t=2, \ldots, T$.

Proof: From (13) we obtain

$$
D_{\mathrm{f}, \infty}^{*}\left(\xi, \xi^{\mathrm{red}}\right)=\sum_{t=2}^{T} \sup _{\left\|x_{t}\right\|_{\infty} \leq 1}\left(\sum_{j \in I_{t}^{\mathrm{red}}} \sum_{i \in J_{t, j}} p_{t}^{i}\left|x_{t}^{i}-\sum_{k \in J_{t, j}} \frac{p_{t}^{k}}{\pi_{t}^{j}} x_{t}^{k}\right|^{r^{\prime}}\right)^{\frac{1}{r^{\prime}}} .
$$

Let $x_{t, s}^{i}, s=1, \ldots, m_{t}$, denote the components of $x_{t}^{i} \in \mathbb{R}^{m_{t}}$ for every $i \in I_{t}$ and $t=2, \ldots, T$. We may continue

$$
\begin{aligned}
D_{\mathrm{f}, \infty}^{*}\left(\xi, \xi^{\mathrm{red}}\right) & =\sum_{t=2}^{T} \sup _{\left\|x_{t}\right\|_{\infty} \leq 1}\left(\sum_{j \in I_{t}^{\mathrm{red}}} \sum_{i \in J_{t, j}} p_{t}^{i} \sum_{s=1}^{m_{t}}\left|x_{t, s}^{i}-\sum_{k \in J_{t, j}} \frac{p_{t}^{k}}{\pi_{t}^{j}} x_{t, s}^{k}\right|^{r^{\prime}}\right)^{\frac{1}{r^{\prime}}} \\
& =\sum_{t=2}^{T} \sup _{\left\|x_{t}\right\|_{\infty} \leq 1}\left(\sum_{s=1}^{m_{t}} \sum_{j \in I_{t}^{\mathrm{red}}} \pi_{t}^{j} \sum_{i \in J_{t, j}} \frac{p_{t}^{i}}{\pi_{t}^{j}}\left|x_{t, s}^{i}-\sum_{k \in J_{t, j}} \frac{p_{t}^{k}}{\pi_{t}^{j}} x_{t, s}^{k}\right|^{r^{\prime}}\right)^{\frac{1}{r^{\prime}}} .
\end{aligned}
$$

Hence, to estimate the filtration distance we have to solve maximum problems of the form

$$
\max \left\{\sum_{i \in J} \lambda_{i}\left|y_{i}-\sum_{k \in J} \lambda_{k} y_{k}\right|^{r^{\prime}}: y \in \mathbb{R}^{|J|}, \max _{i \in J}\left|y_{i}\right| \leq 1\right\}
$$


where $J$ is a given finite index set with cardinality $|J|$ and $\lambda_{i}>0, i \in J$, are given with $\sum_{i \in J} \lambda_{i}=1$. Let $y^{(j)}, j=1, \ldots, 2^{|J|}$, denote the vertices of the polytope $Y:=\{y \in$ $\left.\mathbb{R}^{|J|}: \max _{i \in J}\left|y_{i}\right| \leq 1\right\}$. Any element $y \in Y$ can be represented as convex combination of the vertices, i.e.,

$$
y=\sum_{j=1}^{2^{|J|}} \alpha_{j} y^{(j)}, \text { where } \alpha_{j} \geq 0 \text { and } \sum_{j=1}^{2^{|J|}} \alpha_{j}=1 .
$$

Since the objective function $g(y):=\sum_{i \in J} \lambda_{i}\left|y_{i}-\sum_{k \in J} \lambda_{k} y_{k}\right|^{r^{\prime}}$ in (16) is convex, one obtains

$$
g(y)=g\left(\sum_{j=1}^{2^{|J|}} \alpha_{j} y^{(j)}\right) \leq \sum_{j=1}^{2^{|J|}} \alpha_{j} g\left(y^{(j)}\right) \leq \max _{j=1, \ldots, 2^{|J|}} g\left(y^{(j)}\right) .
$$

Hence, the maximum in (16) is attained at some $y^{*} \in Y$ with $y_{i}^{*} \in\{+1,-1\}$ for all $i \in J$. Let $J^{+} \subseteq J$ and $J^{-} \subseteq J$ denote the index sets, where $y^{*}$ is positive and negative, respectively. Furthermore, let

$$
\lambda^{+}=\sum_{i \in J^{+}} \lambda_{i} \quad \text { and } \quad \lambda^{-}=\sum_{i \in J^{-}} \lambda_{i}
$$

Then we have $\lambda^{+}+\lambda^{-}=1$, and, we obtain

$$
\begin{aligned}
\sum_{i \in J} \lambda_{i}\left|y_{i}^{*}-\sum_{k \in J} \lambda_{k} y_{k}^{*}\right|^{r^{\prime}} & =\sum_{i \in J^{+}} \lambda_{i}\left(1-\lambda^{+}+\lambda^{-}\right)^{r^{\prime}}+\sum_{i \in J^{-}} \lambda_{i}\left(1+\lambda^{+}-\lambda^{-}\right)^{r^{\prime}} \\
& =2 \lambda^{+}\left(1-\lambda^{+}\right)^{r^{\prime}}+2\left(1-\lambda^{+}\right)\left(\lambda^{+}\right)^{r^{\prime}} .
\end{aligned}
$$

If we solve the problem (16) for all $s \in\left\{1, \ldots, m_{t}\right\}, j \in I_{t}^{\text {red }}$ with $J:=J_{t, j}$ and $\lambda_{i}:=\frac{p_{t}^{i}}{\pi_{t}^{j}}$ we get as final estimate for the filtration distance

$$
\begin{gathered}
D_{\mathrm{f}, \infty}^{*}\left(\xi, \xi^{\mathrm{red}}\right) \leq \max _{t=2, \ldots, T} m_{t}^{\frac{1}{r^{\prime}}} \sum_{t=2}^{T}\left(\sum_{j \in I_{t}^{\mathrm{red}}} M_{t, r^{\prime}}^{j}\right)^{\frac{1}{r^{\prime}}}, \quad \text { where } \\
M_{t, r^{\prime}}^{j}:=\max \left\{\frac{2 p\left(\pi_{t}^{j}-p\right)^{r^{\prime}}+2\left(\pi_{t}^{j}-p\right) p^{r^{\prime}}}{\left(\pi_{t}^{j}\right)^{r^{\prime}}}: J \subset J_{t, j}, p=\sum_{i \in J} p_{t}^{i}\right\} .
\end{gathered}
$$

Proposition 3.1 says that the filtration distance between the given process $\xi$ and the reduced one $\xi^{\text {red }}$ only depends on the particular partition structure of the scenarios of $\xi$ and on the choice of representative scenarios of $\xi^{\text {red }}$. The typical cases $r^{\prime}=1$ and $r^{\prime}=2$ are now discussed in more detail. 
The filtration distance for $r^{\prime}=1$ and $r^{\prime}=2$

In case $r^{\prime}=1$ the estimate of the filtration distance is of the form

$$
\begin{aligned}
D_{\mathrm{f}, \infty}^{*}\left(\xi, \xi^{\mathrm{red}}\right) & \leq \max _{t=2, \ldots, T} m_{t} \sum_{t=2}^{T} \sum_{j \in I_{t}^{\mathrm{red}}} M_{t, 1}^{j}, \quad \text { where } \\
M_{t, 1}^{j} & :=\max \left\{\frac{4 p\left(\pi_{t}^{j}-p\right)}{\pi_{t}^{j}} \mid J \subset J_{t, j}, p=\sum_{i \in J} p_{t}^{i}\right\} \leq \pi_{t}^{j} .
\end{aligned}
$$

That allows the following interpretation. For any scenario cluster $J_{t, j}$ defined by the realization $j \in I_{t}^{\text {red }}$, at some stage $t$, the contribution $M_{t, 1}^{j}$ to the total filtration distance depends on the total probability $\pi_{t}^{j}$ of the set $J_{t, j}$ as well as on the partitioning of the probability weights. Note that $M_{t, 1}^{j}$ is always bounded from above by $\pi_{t}^{j}$.

Example:

Let be $J_{t, j}=\left\{k_{1}, \ldots, k_{n}\right\}$ and $p_{t}^{k_{1}}+\cdots+p_{t}^{k_{n}}=\pi_{t}^{j}$.

a) In case $p_{t}^{k_{i}}=p_{t}^{k_{j}}$ for all $i, j=1, \ldots, n$ we obtain $M_{t, 1}^{j}=\frac{n^{2}-1}{n^{2}} \pi_{t}^{j}$ and $M_{t, 1}^{j}=\pi_{t}^{j}$ if $n$ is odd or even, respectively.

b) In case of one dominant probability in the sense that $p_{t}^{k_{j_{0}}}=\lambda \frac{\pi_{t}^{j}}{2}$ with $\lambda \in[1,2]$ we obtain $M_{t, 1}^{j}=\lambda(2-\lambda) \pi_{t}^{j}$. For example, if $\lambda=1.95$ we have $M_{t, 1}^{j}=0.0975 \pi_{t}^{j}$.

Likewise, in case $r^{\prime}=2$ we obtain from Proposition 3.1

$$
\begin{aligned}
D_{\mathrm{f}, \infty}^{*}\left(\xi, \xi^{\mathrm{red}}\right) & \leq \max _{t=2, \ldots, T} m_{t}^{\frac{1}{2}} \sum_{t=2}^{T}\left(\sum_{j \in I_{t}^{\mathrm{red}}} M_{t, 2}^{j}\right)^{\frac{1}{2}}, \quad \text { where } \\
M_{t, 2}^{j} & :=\max \left\{\frac{2 p\left(\pi_{t}^{j}-p\right)}{\pi_{t}^{j}} \mid J \subset J_{t, j}, p=\sum_{i \in J} p_{t}^{i}\right\} \leq \frac{\pi_{t}^{j}}{2} .
\end{aligned}
$$

Figure 3 shows a plot of the objective in problem (17) and (18), respectively, for determining $M_{t, 1}^{j}$ and $M_{t, 2}^{j}$.

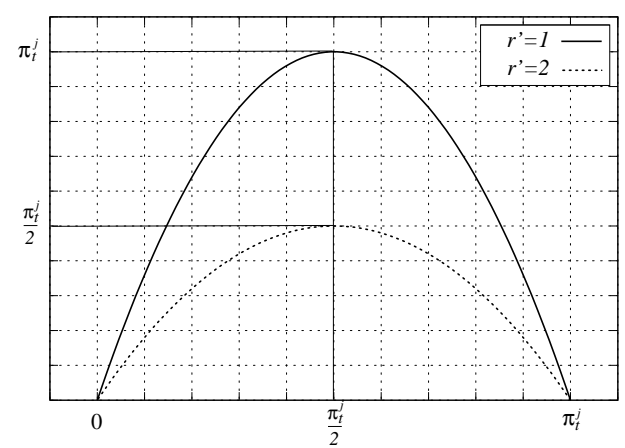

Figure 3: Objective function $f_{t, r^{\prime}}^{j}(p)$ to determine $M_{t, r^{\prime}}^{j}$ in cases $r^{\prime}=1$ and $r^{\prime}=2$. 


\section{Scenario tree reduction in multistage models}

In this section we are going to describe a simple scenario reduction algorithm which is based on recursive single node reduction. For a given tree structure of $\xi$, the criterion (9), the representation (14), and the estimate (15) suggest the following reduction strategy of $\xi$ given some tolerance $\varepsilon>0$.

\section{Algorithm (Single node reduction)}

\section{[nitialization]}

The reduction procedure is initialized by starting from the initial process, i.e., by setting

$$
\begin{aligned}
I_{t}^{\mathrm{red}} & :=I_{t} \text { for all } t=1, \ldots, T, \\
I_{t, i}^{\mathrm{red}} & :=I_{t, i} \text { for all } t=1, \ldots, T-1, i \in I_{t}, \\
J_{t, j} & :=\{j\} \text { for all } t=1, \ldots, T, j \in I_{t}, \\
q_{t}^{j} & :=p_{t}^{j} \text { for all } t=1, \ldots, T, j \in I_{t}, \\
\varepsilon_{\text {appr }} & :=0,
\end{aligned}
$$

where $\varepsilon_{\text {appr }}$ denotes the approximation error of the reduction process.

\section{[Node selection]}

The node selection aims at determining an acceptable pair of nodes. A pair $(t, j)$ and $(\tau, i)$ is called acceptable whenever $t=\tau, i \neq j$, the unique predecessors of both nodes coincide, and, simultaneously, the approximation error is small enough. To this end, we search for some $(t, j) \in\{2, \ldots, T\} \times I_{t}^{\text {red }}$ such that there exists $i \in I_{t}^{\text {red }}$ with $i \neq j$, $i, j \in I_{t-1, k}^{\text {red }}$ for some $k \in I_{t-1}^{\text {red }}$ and such that the pair $(t, j)$ and $(t, i)$ of nodes satisfies the estimate

$$
\begin{aligned}
& \varepsilon_{\text {step }} \leq \varepsilon-\varepsilon_{\text {appr }}, \quad \text { where } \\
& \varepsilon_{\text {step }}:=w_{1}\left(q_{t}^{i}\right)^{\frac{1}{r}}\left|\xi_{t}^{i}-\xi_{t}^{j}\right|+w_{2} \frac{\left(2 q_{t}^{i}\left(q_{t}^{j}\right)^{r^{\prime}}+2\left(q_{t}^{i}\right)^{r^{\prime}} q_{t}^{j}\right)^{\frac{1}{r^{\prime}}}}{q_{t}^{i}+q_{t}^{j}} .
\end{aligned}
$$

If such a pair cannot be found, go to the termination step, otherwise continue with the following reduction step.

\section{[Reduction]}

In this step we perform the node reduction according to the selection of acceptable nodes before. We adjust the relevant index sets and probabilities of the scenario tree by enlarging the set $J_{t, j}$, reducing the set $I_{t}$, updating the successor information and changing the probabilities. The new index sets and probabilities are given by

$$
\begin{aligned}
J_{t, j} & :=J_{t, j} \cup J_{t, i}, \\
I_{t}^{\text {red }} & :=I_{t} \backslash\{i\}, \\
I_{t, j}^{\text {red }} & :=I_{t, j} \cup I_{t, i}, \\
q_{t}^{j} & :=q_{t}^{j}+q_{t}^{i} .
\end{aligned}
$$


Finally, the approximation error is updated by

$$
\varepsilon_{\text {appr }}:=\varepsilon_{\text {appr }}+\varepsilon_{\text {step }},
$$

and the iteration is continued by a new node selection step.

\section{[Termination]}

Whenever the termination step is reached all relevant index sets giving the structure of the reduced scenario tree process are stored as $I_{t}^{\text {red }}, I_{t, j}^{\text {red }}$ and $J_{t, j}$ (cf. Section 3 ). It remains to define the (node) probabilities by

$$
\pi_{t}^{j}:=q_{t}^{j} \quad \text { for all } \quad t=1, \ldots, T, j \in I_{t}^{\text {red }} .
$$

The process $\xi^{\text {red }}$ is well-defined now by the given index sets and probabilities.

We conclude this section with some comments on the above algorithm. In fact, we obtain for the probabilities of the scenario tree process $\xi^{\text {red }}$ that

$$
\pi_{t}^{j}=\sum_{i \in J_{t, j}} p_{t}^{i} \quad \text { for all } \quad t=1, \ldots, T, j \in I_{t}^{\mathrm{red}}
$$

Moreover, the approximation error between the initial scenario tree process and the reduced one can be bounded by $\varepsilon_{\text {appr }}$. More precisely, it holds

$$
w_{1}\left\|\xi-\xi^{\mathrm{red}}\right\|_{r}+w_{2} D_{\mathrm{f}, \infty}^{*}\left(\xi, \xi^{\mathrm{red}}\right) \leq \varepsilon_{\mathrm{appr}},
$$

which is a direct consequence of (19) and the triangle inequality for both the $L_{r}$-distance and the filtration distance $D_{\mathrm{f}, \infty}^{*}$. Note that the reduction algorithm also can be easily performed with respect to only one distance by setting the weighting factors $w_{1}=0$ and $w_{2}=0$, respectively. If we define $w_{1}=0$ in condition (19) the term controlling the $L_{r}$-distance disappears. On the other hand, when using $w_{2}=0$ the filtration term disappears and, hence, the reduction is only performed with respect to the $L_{r}$-distance.

\section{$5 \quad$ Numerical experience}

Finally, we report on some preliminary numerical experience for scenario tree reduction in multistage stochastic programs. For testing the single node reduction algorithm of the previous section, we consider a stochastic optimization model for electricity portfolios of a German municipal power company. The portfolio consists of the own (thermal) electricity production, the spot market contracts, supply contracts and electricity futures. For details of the optimization model we refer to [5]. It takes into account the stochastic nature of the input parameters for every hour of the underlying time horizon, namely, the electricity demand, the heat demand, the EEX spot prices, and base and peak future prices (for each month). Here, we focus on the input scenario tree process and assume that it is obtained by the scenario tree generation method of [9]. Since the future prices are considered as fair prices and can be derived from the spot prices, the input scenarios correspond to a trivariate time discrete stochastic input process whose components are electricity demand, heat demand, and (EEX) spot prices. 
For our purposes a generated scenario tree process $\xi$ is singled out and reduced by the algorithm in Section 4 until a prescribed number of nodes is reached. To study, in particular, the impact of the filtration distance, scenario trees $\xi^{\text {red }}$ are computed by the single node reduction algorithm, where the reduction is done with respect to the $L_{r}$-distance and the $D_{\mathrm{f}, \infty}^{*}$-distance separately as well as with respect to the sum of both distances as advised by the stability analysis of Section 2 .

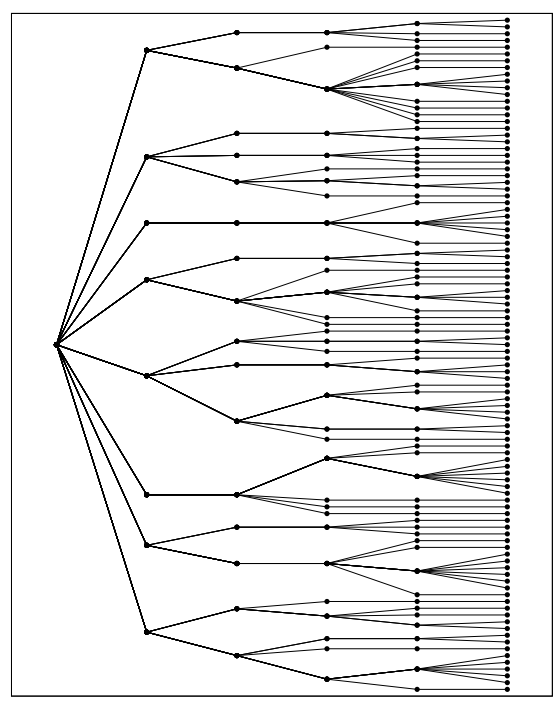

Figure 4: Structure of the trivariate initial scenario tree $\xi$ serving as input for the single node reduction algorithm.

Due to modeling reasons the input scenario tree exhibits a monthly branching structure. For our numerical test we considered a time horizon of 6 months which correspond, hence, to 6 stages of the stochastic program. In order to cope with this monthly structure, each component of the scenarios (corresponding to electricity demand, heat demand or spot prices) is represented by 6 vectors, where each vector contains the inputs of one month in hourly discretization. The tree structure of the input process is illustrated in Figure 4.

\begin{tabular}{llccccccc}
\hline Nodes & Reduction & \multicolumn{9}{c}{ Nodes (per Stage) } & Scenarios \\
& w.r.t. & 1 & 2 & 3 & 4 & 5 & 6 & \\
\hline 150 & $L_{r}$ only & 1 & 8 & 15 & 23 & 40 & 63 & 63 \\
150 & $L_{r}$ and $D_{\mathrm{f}, \infty}^{*}$ & 1 & 8 & 16 & 23 & 41 & 61 & 61 \\
150 & $D_{\mathrm{f}, \infty}^{*}$ only & 1 & 8 & 16 & 24 & 41 & 60 & 60 \\
\hline 100 & $L_{r}$ only & 1 & 6 & 12 & 17 & 25 & 39 & 39 \\
100 & $L_{r}$ and $D_{\mathrm{f}, \infty}^{*}$ & 1 & 8 & 15 & 19 & 25 & 32 & 32 \\
100 & $D_{\mathrm{f}, \infty}^{*}$ only & 1 & 8 & 16 & 20 & 26 & 29 & 29 \\
\hline
\end{tabular}

Table 1: Structure of the scenario tree processes $\xi^{\text {red }}$ obtained by the single node reduction algorithm starting from the input tree containing 221 nodes and terminating with trees containing 150 and 100 nodes, respectively. 

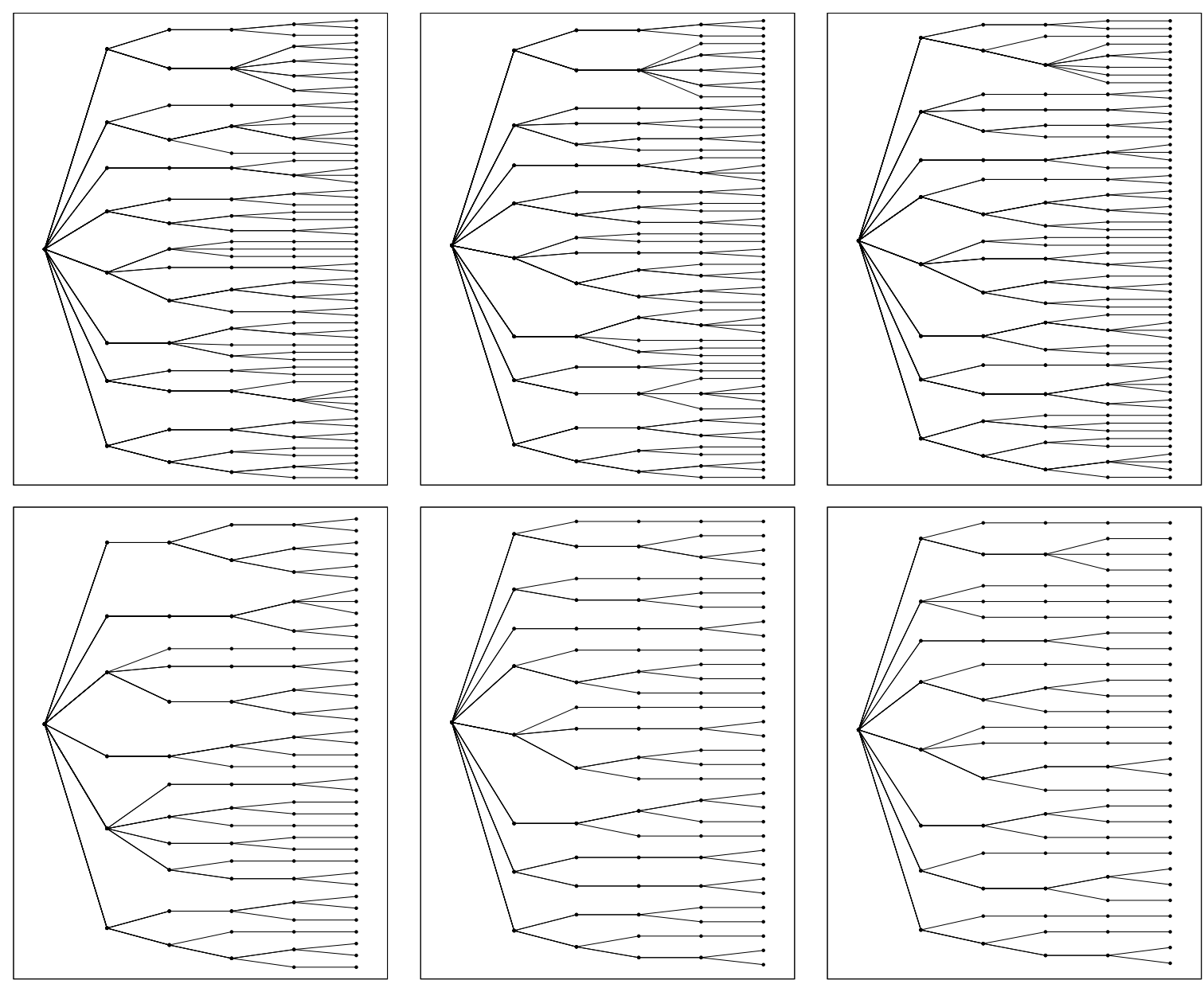

Figure 5: Illustration of the reduced (trivariate) scenario trees $\xi^{\text {red }}$. The trees are obtained by the single node reduction algorithm until 150 (above) and 100 (below) remaining nodes are reached. The reduction is carried out with respect to the $L_{r^{-}}$ distance only (left), both the $L_{r^{-}}$and the $D_{\mathrm{f}, \infty}^{*}$-distance (middle), and the $D_{\mathrm{f}, \infty}^{*}$-distance only (right).

Figure 5 illustrates the results of the scenario tree reduction by applying the single node reduction algorithm until 150 and 100 nodes remain, respectively. They show that the filtration distance influences the structure of the reduced scenario trees noticeably. The incorporation of the filtration distance leads to a larger number of remaining scenarios in both cases. The opposite effect appears when using the $L_{r}$-distance only.

\section{Conclusions}

Summarizing our theoretical arguments and preliminary numerical experience indicates that the incorporation of the filtration distance into the reduction of scenario trees is indispensable. This implies, in particular, that deleting scenarios in input trees for multi-stage models according to the methodology presented in $[3,7,8]$ is not appropriate as the information (filtration) structure is not taken into account. The numerical 
results in Section 4 are obtained by a simple straightforward strategy of reducing single nodes recursively. But, the estimates (14) and (15) for the $L_{r^{-}}$and filtration distance offer further potential for algorithmic extensions.

\section{Acknowledgement:}

This work was supported by the DFG Research Center MATheon "Mathematics for key technologies" in Berlin and by the BMBF under the grant 03SF0312E.

\section{References}

[1] Casey, M.; Sen, S.: The scenario generation algorithm for multistage stochastic linear programming, Math. Oper. Res. 30 (2005), 615-631.

[2] Dupačová, J.; Consigli, G.; Wallace, S. W.: Scenarios for multistage stochastic programs, Ann. Oper. Res. 100 (2000), 25-53.

[3] Dupačová,J.; Gröwe-Kuska, N.; Römisch, W.: Scenario reduction in stochastic programming: An approach using probability metrics, Math. Program. 95 (2003), 493-511.

[4] Eichhorn, A.; Römisch, W.: Polyhedral risk measures in stochastic programming, SIAM J. Optim. 16 (2005), 69-95.

[5] Eichhorn, A.; Römisch, W.: Mean-risk optimization models for electricity portfolio management, Proceedings of PMAPS 2006 (Probabilistic Methods Applied to Power Systems), Stockholm (Sweden), 2006.

[6] Eichhorn, A.; Römisch, W.: Stability of multistage stochastic programs incorporating polyhedral risk measures, Optimization (to appear).

[7] Heitsch, H.; Römisch, W.: Scenario reduction algorithms in stochastic programming, Comp. Optim. Appl. 24 (2003), 187-206.

[8] Heitsch, H.; Römisch, W.: A note on scenario reduction for two-stage stochastic programs, Oper. Res. Let. 35 (2007), 731-736.

[9] Heitsch, H.; Römisch, W.: Scenario tree modeling for multistage stochastic programs, Math. Program. (to appear).

[10] Heitsch, H.; Römisch, W.: Stability and scenario trees for multistage stochastic programs, submitted to Stochastic Programming - The State of the Art (G. Infanger ed.).

[11] Heitsch, H.; Römisch, W.; Strugarek, C.: Stability of multistage stochastic programs, SIAM J. Optim. 17 (2006), 511-525.

[12] Henrion, R.; Küchler, C.; Römisch, W.: Scenario reduction in stochastic programming with respect to discrepancy distances, Comp. Optim. Appl. (to appear).

[13] Henrion, R.; Küchler, C.; Römisch, W.: Discrepancy distances and scenario reduction in two-stage stochastic integer programming, Preprint 397, DFG Research Center MathEON "Mathematics for key technologies", 2007 and submitted to Journal of Industrial and Management Optimization. 
[14] Hochreiter, R.; Pflug, G. Ch.: Financial scenario generation for stochastic multi-stage decision processes as facility location problems, Ann. Oper. Res. 152 (2007), 257-272.

[15] Høyland, K.; Kaut, M.; Wallace, S.W.: A heuristic for moment-matching scenario generation, Comp. Optim. Appl. 24 (2003), 169-185.

[16] Kuhn, D.: Generalized bounds for convex multistage stochastic programs, Lecture Notes in Economics and Mathematical Systems, Vol. 548, Springer, Berlin, 2005.

[17] Kuhn, D.: Aggregation and discretization in multistage stochastic programming, Math. Program. 113 (2008), 61-94.

[18] Pennanen, T.: Epi-convergent discretizations of multistage stochastic programs via integration quadratures, Math. Program. (to appear).

[19] Rachev, S. T.; Rüschendorf, L.: Mass Transportation Problems, Vol. I and II, Springer, Berlin 1998.

[20] Römisch, W.: Stability of Stochastic Programming Problems, in: Stochastic Programming (A. Ruszczyński and A. Shapiro eds.), Handbooks in Operations Research and Management Science, Volume 10, Elsevier, Amsterdam 2003, 483-554.

[21] Römisch, W.; Wets, R. J-B: Stability of $\varepsilon$-approximate solutions to convex stochastic programs, SIAM J. Optim. 18 (2007), 961-979.

[22] Ruszczyński, A.; Shapiro, A. (Eds.): Stochastic Programming, Handbooks in Operations Research and Management Science, Volume 10, Elsevier, Amsterdam 2003. 